\title{
Experimental Investigation and Optimization for the Effective Parameters in the Laser Direct Structuring Process
}

\author{
Bassim Bachy ${ }^{\mathrm{a}, 1}$ and Jörg Franke ${ }^{2}$ \\ ${ }^{1,2}$ Institute for Factory Automation and Production Systems, \\ Friedrich-Alexander-University Erlangen-Nüremberg, Nüremberg, Germany \\ ${ }^{a}$ E-mail:Bassim.Bachy@faps.fau.de
}

\begin{abstract}
The laser direct structuring (LDS) and the molded interconnect devices (MID) processes become more popular in the electronics industry. In order to produce micro MID products with highest circuit lines quality, it is very important to understand the complex relationship among the laser direct structuring parameters, the dimensions of the groove at the substrate surface, the profile of this groove, the width of the interactive zone (circuit line width) and space between two circuit lines (pitch). To reveal this relationship, experiments on the polymer plate (polyphthalamide PPA, with glass fiber) have been carried out in this work. It was found that the effect of the laser speed and laser power on the groove profile and dimensions are greater than the effect of the laser frequency, moreover the metallization profile depends on the groove profile which again depends on the LDS parameters. The dimensions of the resolidification groove edge including the width and height must be reduced to lowest possible value, in order to reduce the metallization defects.
\end{abstract}

DOI: $10.2961 / \mathrm{jlmn} .2015 .02 .0018$

Keywords: Laser process, molded interconnected devices process, laser direct structuring process, Microstructure.

\section{Introduction}

In the last few years the laser direct structuring technique (LDS) in conjunction with molded interconnected devices (MID) have been used to produce the electronic circuit board with high accuracy. Most of the previous work introduced the MID process as an important new technique in the electronic products such as electronic board with fine or high resolution circuit lines and concluded that it is possible to reduce the circuit line thickness. This process was developed by LPKF Laser \& Electronics $\mathrm{AG}$, a manufacturer of industrial laser technology headquartered in Garbsen, Germany [1]. In general, the LDS process consists of three steps: injection molding and laser structuring followed by metallization for conductive patterns.

In the previous work [2], the MID process was introduced as an important and accurate method to produce the 3 dimensional electronics parts (3D). This paper gives an overview on the advantage of galvanic plating for 3DMIDs, as well as the differences in the plating processes (metallization) between the galvanic and the chemical precipitation.

Theoretical works including simulations by ANSYS in conjunction with experimental works have been conducted by B. Bachy and J. Franke for the LDS in the MID process [3]. In this paper the effect of the laser parameters such as laser speed and laser power on the groove width and depth as well as the quality of the groove edges have been studied. The researchers concluded that, the quality of laser groove increase with decreasing the groove edges dimensions.
Another study has been carried out by L. Romoli et al [4]. This study deals with an innovative technique concerning $\mathrm{CO}_{2}$ laser in order to create $3 \mathrm{D}$ cavities for the polymer Polymethylmethacrylat (PMMA). In this work, the effects of the laser parameter such as laser power and laser scan speed on the laser groove width and depth have been studied. The researchers concluded that, the removal or the groove depth increase when the number of layers increases.

The relationship among laser fluence and the ablation depth and rate for different materials has been studied by $\mathrm{G}$ Raciukaitis [5] and E. Spyratou [6]. These works include experimental works to study the effect of the laser fluence on the depth and the rate of the metal removal rate from the surface of the tested material.

An experimental work has been reported for the laser micromachining by C.R. Mendonca et al [7]. In this experimental study, the effect of some laser parameters such as the laser power and the laser scan speed on the depth of the laser groove have been investigated. In this paper, the researchers studied the effect of the laser power on the metal removal rate from the surface of the $\mathrm{MEH}-$ PPV polymer.

The influence of laser beam polarization in the laser micromachining of molybdenum has been studies by Rémi T. [8]. In this work, the relationship between the laser beam and the quality of the laser machining has been instigated and the researchers concluded that the quality of the laser cutting edge will be increased by changing the laser polarization.

F. Luo, et al. [9], have been studied the effect of the laser scanning speed and the gaseous environment including air, O2, N2 and Ar on the micro/nanostructures 
formed by a nanosecond laser for the stainless steel. The researchers concluded that the, the dimensional feature, the chemical composition and the surface color for the structure are greatly affected by the laser scanning speed and the gaseous environment. This study provides new promising applications for laser in field of the surface marking and code identifying.

In the present work, experimental tests have been carried out to study the effect of the laser direct structuring (LDS) parameters on the profile and the dimensions of the micro groove. The most important LDS parameters including laser scan speed, laser power, laser frequency and the width of the space between the two circuit lines (Pitch) have been studied in this work. It is very important to mention that the quality of the MID product is depending on the most important steps which are laser direct structuring step and the metallization step. On the other hand, the quality of the laser direct structuring step is depending on the dimensions and profile of the laser groove. For each LDS parameters we have different groove dimensions and profile, and the best groove profile and dimensions can be achieved only by using the suitable and the optimum laser parameters, so that depending on the groove profile, a circuit lines with high quality or minimum of defects can be produced. Figure 1 (a), shows the 3D laser microscope scan for the groove profile at the surface of the polymer after the LDS process. The 2D cross sectional area for the groove is shown in figure 1 (b). From this figure, we can see the description for the important dimensions including groove depth and width, groove edge width and height and the width of the interactive zone for this groove as well as the groove profile.

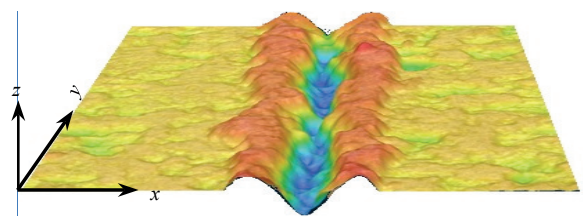

(a) 3D Laser scan image for the groove profile.

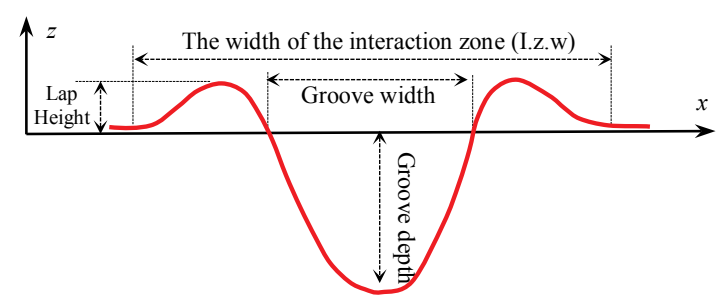

(b) The description for the important groove dimensions.

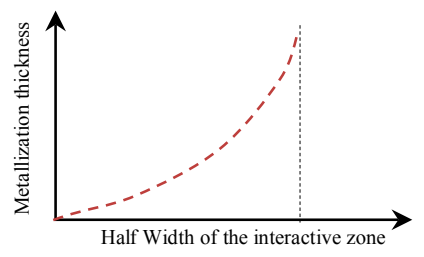

(c) The metallization thickness.

Fig.1 The groove profile and the metallization thickness.

During the metallization all the interactive width will be covered by the $\mathrm{Cu}$ metal, not only the width of the groove channel, with different metallization thickness. The maximum value of this thickness is at the groove center, while the minimum at the edge of the effective zone, as shown in figure 1 (c). In fact, there is a very important relationship between the metallization thickness and the profile of the groove after the LDS process. The above behavior for the groove profile and the metallization profile will be changed when two lines create together with specific distance between them. This is due to the heat interaction between the two grooves, which may be lead to produce defect between the two grooves (circuit lines). This is absolutely depending on the distance between the two lines (pitch), so that one of the most important aims of the present work is to study this behavior and its relationship with the other LDS parameters, this helps to find the minimum linelpitch.

\section{The Experimental Work}

A polymer plates with dimensions of $60 \times 60 \times 2 \mathrm{~mm}$. have been used in the experimental works. And the material is VESTAMID® HT plus LDS 3031 black, which is a mineral reinforced polyphthalamide (PPA) with glass fiber. This compound is designed to be used in the production of three dimensional interconnect devices by laser direct structuring (according to the LPKF LDS technology) [9]. A laser machine provided a Nd:YAG laser with $1064 \mathrm{~nm}$ wavelength, power in the range of form 1 to $17 \mathrm{~W}$, laser beam diameter $65 \mu \mathrm{m}$, the maximum frequency $200 \mathrm{kHz}$ and the maximum pulse duration 23,7 ns have been used to perform the tests.

In order to study the relationship among the LDS parameters and the minimum line space, two circuit lines have been created in this experiment tests with different proposal space $(P s)$ width between these two lines, as shown in figure 2 . The values of the $P S$ distance have been supposed as a function for laser beam diameter $(2 \mathrm{Xd}, 3 \mathrm{Xd}$, $4 \mathrm{Xd}$ ), where $\mathrm{d}$ is the laser beam diameter. For each $P s$, different value of the LDS parameters have been used including laser powers of 3, 6, 9 and $12 \mathrm{~W}$, the laser speed of $1000,1300,1600,1900$ and $2200 \mathrm{~mm} / \mathrm{s}$ and finally the laser frequency of 70, 90, 110 and $130 \mathrm{kHz}$.

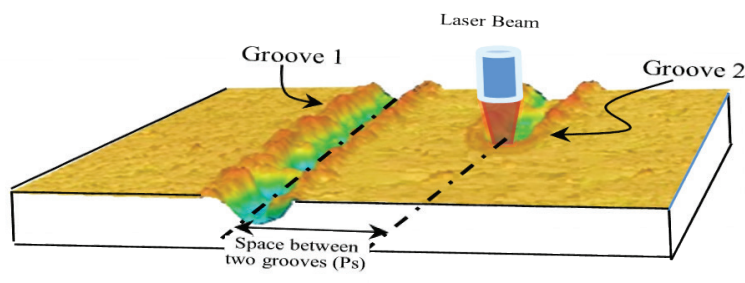

Fig.2 Schematic of experimental test.

Figure 3, shows the flowchart for the experimental tests. The total numbers of the experimental tests carried out in this work are 240. 3D laser scanning microscope (Keyence) has been used to examine the laser effect on the surface of the polymer and measure the groove dimensions and profile. These measurements have been performed after the LDS process and after the metallization process to examine the metallization profile; furthermore a comparison between these two profiles (groove and metallization profiles) has been carried out. 


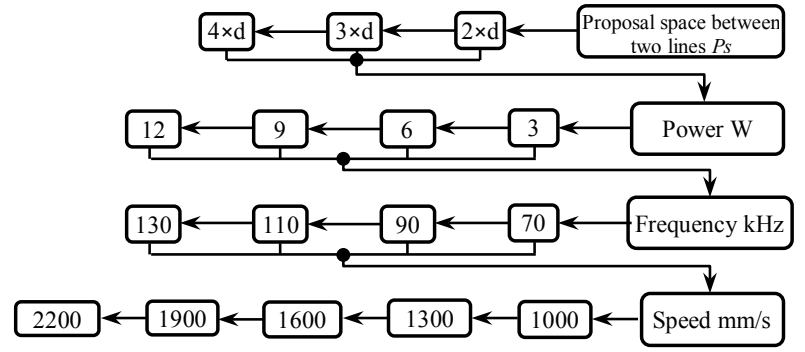

Fig. 3 The experimental tests.

\section{Results and Discussion}

\subsection{The effect of LDS parameters on the groove dimensions}

The groove characteristics including, groove width, groove depth, interaction width and groove edge dimensions, as well as the minimum space between the two circuit lines, have been measured after the LDS process using the laser microscope scan. From the experimental tests of the LDS process, which are conducted under different LDS parameters, the effect of these parameters will be investigated in this part of the work for the proposal space value $(P s)$ equal to $3 \times \mathrm{d}(195 \mu \mathrm{m})$.

The effect of the laser speed on the groove depth at different laser power and laser frequency is presented in figure 4 (a). It can be observed that the groove depth decreases with laser speed and increases with laser power. In fact the relationship between the groove depth and laser speed affected by the amount of the laser power and laser frequency, as shown in figure 4 (a) and (b). The increasing in the laser power leads to increase the heat input heat to the surface of the polymer, then the material removing rate from the surface of the polymer will be increased as result the groove depth and width will be increased.

A very important phenomenon can be noted from figure 4 (b), where at laser power of $3 \mathrm{~W}$, laser frequency of $130 \mathrm{kHz}$ and laser speed more than $1600 \mathrm{~mm} / \mathrm{s}$, the groove depth is 0 , this means at low laser power, high laser speed and high frequency, there is no groove at the polymer surface. In fact, under such these LDS parameters the amount of the heat flux is high enough to melt the polymer surface but not enough for the vaporization this molten polymer from the surface, as a result, like puffiness or blown occurs on the polymer surface. Also, figure 4 (b) shows the effect of the laser frequency on the groove depth, it can be noted that the groove depth decrease with laser frequency.

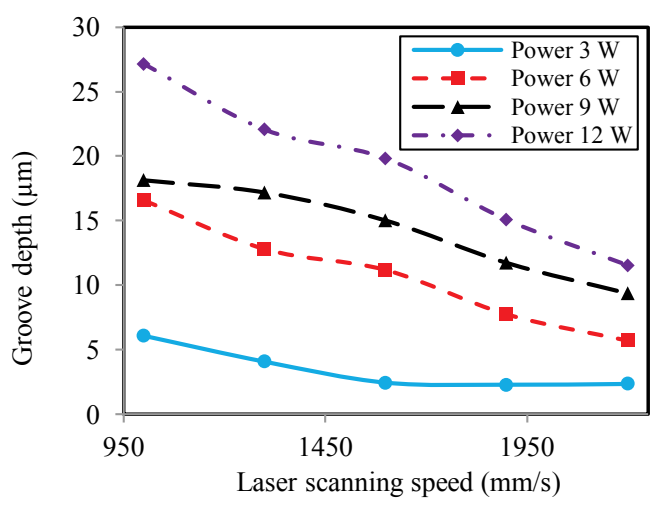

(a) At laser frequency of $90 \mathrm{kHz}$.

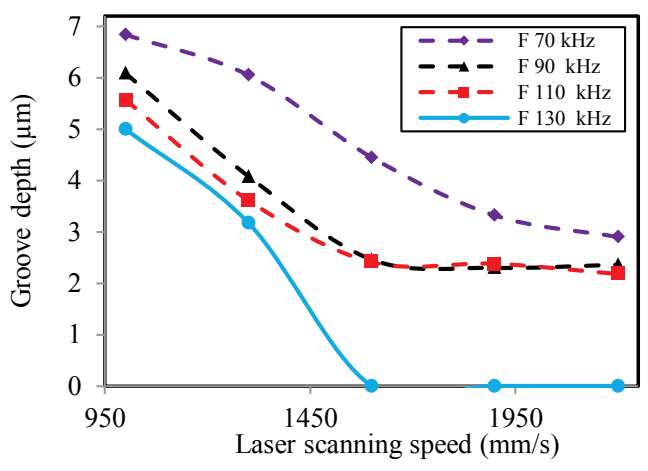

(b) At laser power of $3 \mathrm{~W}$

Fig. 4 The effect of laser speed on the groove depth at different laser power and frequency.

Another important dimension is the groove width or the width of the ablation material from the surface of the polymer during the LDS process. Figure 5, shows the effect of the laser speed on the groove width at different laser power and different laser frequency. It can be seen that the effect of LDS parameters on the groove width is the same as on the groove depth. Also, it can be seen that the groove width is 0 under the LDS conditions including laser power of $3 \mathrm{~W}$, laser speeds of more than $1600 \mathrm{~mm} / \mathrm{s}$ and the laser frequency of $130 \mathrm{kHz}$. Under these conditions, there is no groove at the surface of the polymer; this is due to the blown phenomena as mentioned previously.

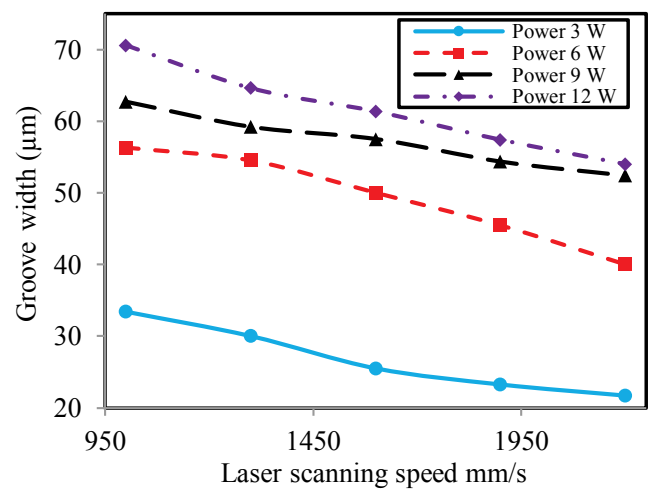

(a) At laser frequency of $70 \mathrm{kHz}$.

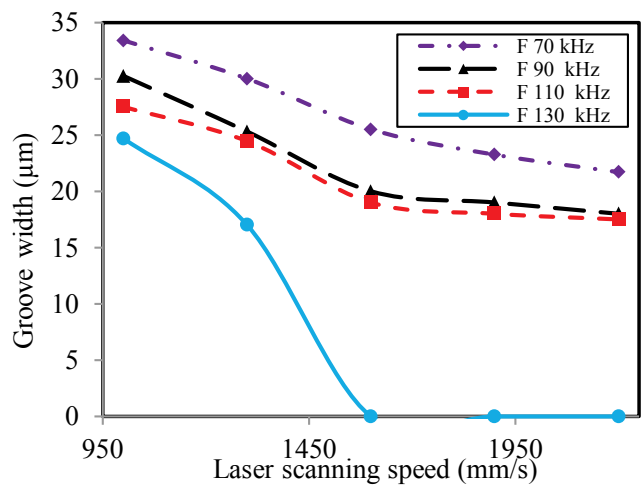

(b) At laser power of $3 \mathrm{~W}$.

Fig. 5 The effect of laser speed on the groove width at different laser power and frequency. 
The quality of the circuit lines depends on the width of the interactive zone because during the metallization process the $\mathrm{Cu}$ metal covers and coats the entire width of the effective zone as mentioned previously, with profile depends on the groove profile which again depends on the LDS parameters. Hence it is important to understand the relationship between the LDS parameters and the width of the interactive zone, this relationship is presented in figure 6 . It can be seen that the width of the interactive zone at constant laser power and constant frequency decreases when the laser speed increases, but the slope of this relationship depends on the laser power and frequency. Furthermore it can be noted that the width of the interactive zone increases with the laser power at fixed laser speed and frequency.

The maximum width of the interactive zone (circuit line) of $142.46 \mu \mathrm{m}$ can be achieved under the LDS parameters of $12 \mathrm{~W}$ laser power, $1000 \mathrm{~mm} / \mathrm{s}$ laser speed and $70 \mathrm{kHz}$ laser frequency, as shown in figure 6 (a), while the minimum width of the interactive zone of $48.5 \mu \mathrm{m}$ under $3 \mathrm{~W}$ laser power, $2200 \mathrm{~mm} / \mathrm{s}$ laser speed and $130 \mathrm{kHz}$ laser frequency, as shown in figure 6 (b). In order to produce a circuit line with width in range of from 48.5 to $142.46 \mu \mathrm{m}$, many different possible sets of the LDS parameters (not only one) can be used to produce it, this gives a high flexibility to use the LDS parameters to produce a certain width of the circuit line.

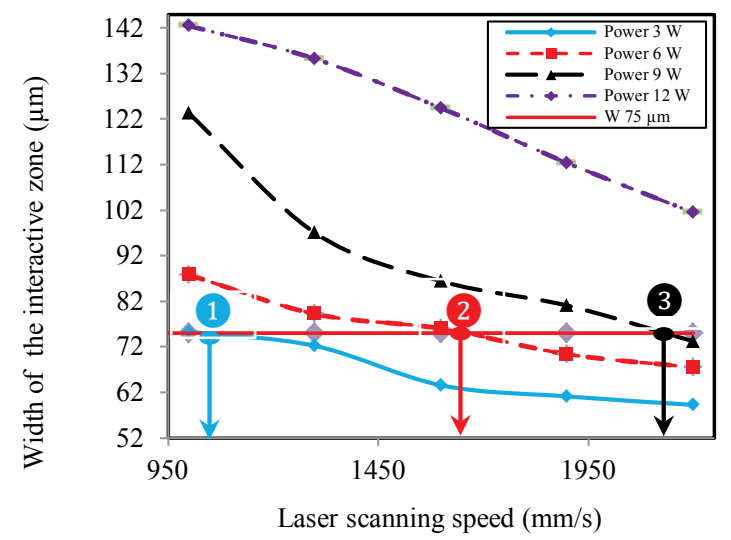

(a) At laser frequency of $70 \mathrm{kHz}$.

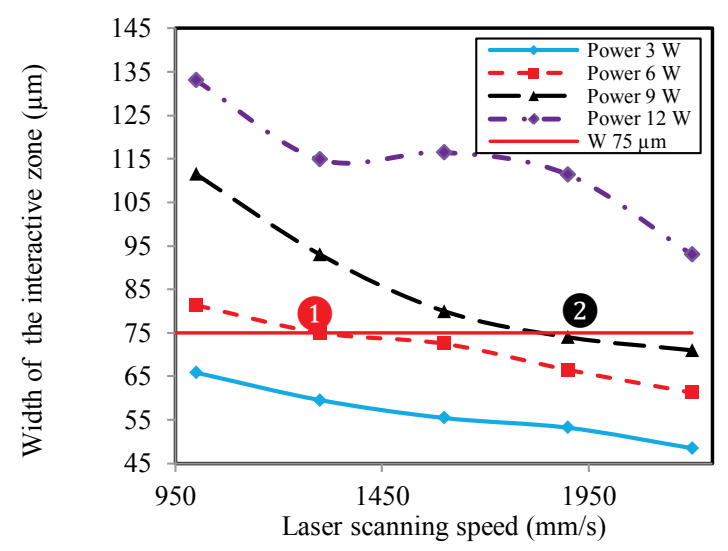

(b) At laser frequency of $130 \mathrm{kHz}$.

Fig. 6 The effect of laser speed on the width of the interaction zone at different laser power and frequency.
Another important effect for the laser frequency on the interactive width shown in figure 7 , it can be seen that the interactive width decreases with laser frequency. Furthermore at low laser power of $3 \mathrm{~W}$ and laser frequencies 70 and $130 \mathrm{kHz}$, the difference in the interactive width can be seen clearly, but this difference is approximately negligible in comparison with laser frequencies 90 and $110 \mathrm{kHz}$, as shown in figure 7 (a). Additionally, at laser power of $6 \mathrm{~W}$ and laser frequency in range of from 70 to $130 \mathrm{kHz}$, it can be seen that there is no high effect for the laser frequency on the interactive width, as shown in figure 7 (b).

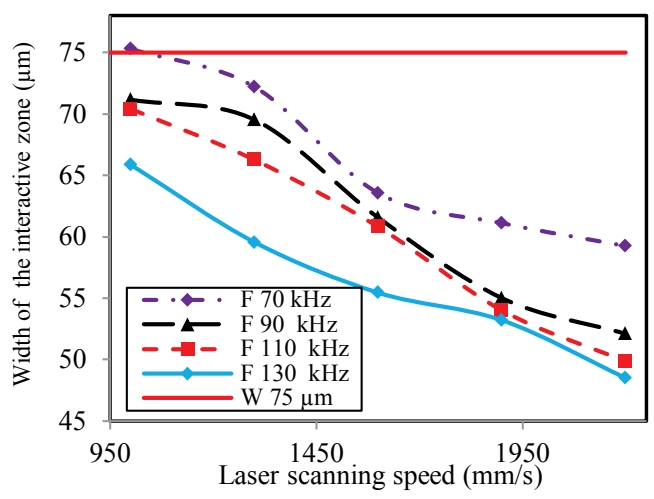

(a) At laser power of $3 \mathrm{~W}$.

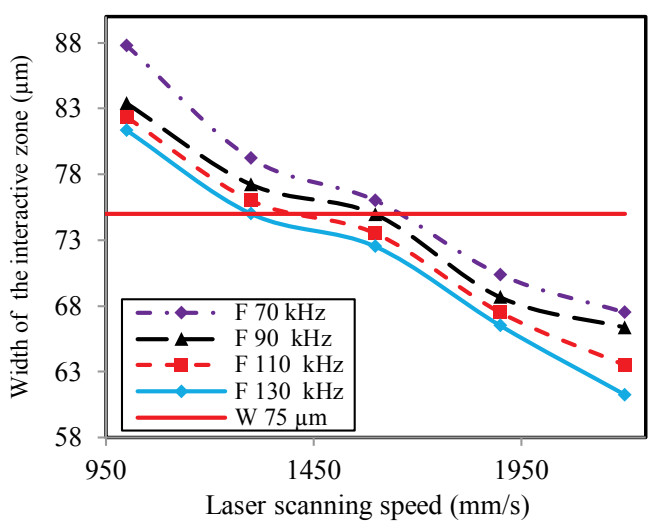

(b) At laser power of $6 \mathrm{~W}$.

Fig.7 The effect of laser speed on the width of the interaction zone at different laser frequency and power.

It can be concluded from figures 6 and 7 that there is high flexibility by using the LDS parameters to produce a specific interactive width (circuit line), for example, a interactive width of $75 \mu \mathrm{m}$ can be produced under the LDS parameters as shown in Table 1.

Table 1 The sets LDS parameters can be used to create a fixed interactive zone width of $75 \mu \mathrm{m}$

\begin{tabular}{|c|c|c|c|}
\hline Cases & & Laser power $\mathrm{W}$ & Laser speed $\mathrm{mm} / \mathrm{s}$ \\
\hline Case 1 & 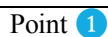 & 3 & 1050 \\
\hline Laser frequency & Point 2 & 6 & 1650 \\
\hline of $70 \mathrm{kHz}$ & Point 3 & 9 & 2125 \\
\hline Case 2 & Point 1 & 6 & 1300 \\
\hline $\begin{array}{c}\text { Laser frequency } \\
\text { of } 130 \mathrm{kHz}\end{array}$ & Point 2 & 9 & 1825 \\
\hline
\end{tabular}

Figure 7, can be used also to suggest the sets of the optimal LDS parameters which can be used to create circuit line with width of $75 \mu \mathrm{m}$. It can be seen that at each laser power there are a different points that determine the LDS 
parameters group. For example at laser power of $3 \mathrm{~W}$, can be used only one point for the LDS parameters, as shown in figure 7 (a). While at laser power of $6 \mathrm{~W}$, shown in figures 7 (b), there are four points for the LDS parameters.

The 3D surface for the relationship among the interactive width and the LDS parameters, namely speed, laser power and laser frequency, shown in the figure 8. It can be seen the three groups of the LDS parameters that can be used to produce a circuit line with width of $75 \mu \mathrm{m}$. The intersection line between the horizontal plane of $75 \mu \mathrm{m}$ and the surfaces for the laser powers of 3, 6, and $9 \mathrm{~W}$ represents these groups. It is very important to mention that at laser power of $12 \mathrm{~W}$, an interactive width of $75 \mu \mathrm{m}$ cannot produce under the LDS parameters including laser speed in range of from 1000 to $2200 \mathrm{~mm} / \mathrm{s}$ and laser frequency in range of from 70 to $130 \mathrm{kHz}$, while at laser powers of 9 and $6 \mathrm{~W}$, many groups for the LDS parameters can be used, and at laser power of $3 \mathrm{~W}$ a few groups.

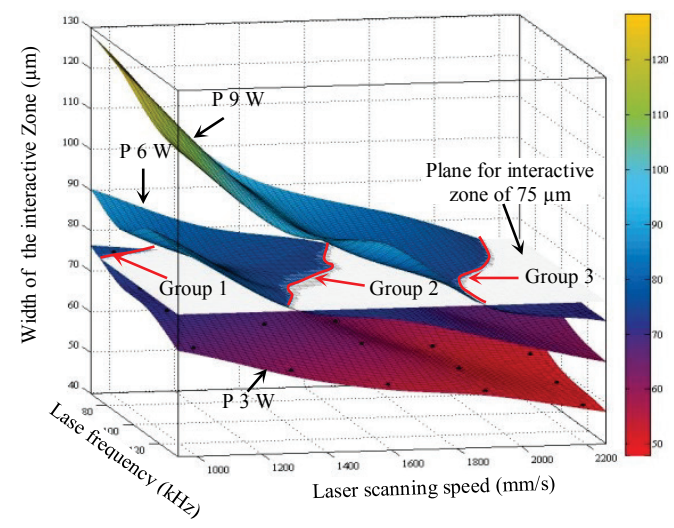

Fig.8 The effect of LDS parameters on the interactive width.

The micro MID products require a minimum groove dimensions namely interactive width (circuit line width), space between two grooves (pitch) as well as the groove edge dimensions. Previously the relationship between the LDS parameters and the interactive width has been studied. Now it is very important to study the effect of the LDS parameters on the minimum space between two circuit lines and to estimate or make it under the control. There is a very important relationship between the interactive zone width and the minimum distance achieved between the two circuit lines. The relationship among the proposal distance $(P s)$ of the two grooves, interactive zone width (I.z.W) and the minimum space $(M S)$ or distance between two circuit lines can be calculated by using equation (1).

$M s=P s-I . z . w$

Equation 1 can be used when; there is no interaction between the heat transfers for the two grooves during the LDS process. This effect not occurs under high Ps, but it is a serious problem for the micro MID products, which require low PS. Actually this effect can be removed or reduced by changing the sequence of the laser structuring for the circuit lines to give enough time for cooling and then prevent the heat interaction between the grooves.

It has been found that at the minimum distances $(M s)$ in the range of from 50 to $60 \mu \mathrm{m}$, the circuit lines after the metallization may be subjected to the damage, where the metal covers entire interactive width for the two grooves and the distance between them, as presented in figure 9 (a), it shows the results for an experimental test performed under the LDS parameters including laser power of $12 \mathrm{~W}$, laser speed of $1000 \mathrm{~mm} / \mathrm{s}$ and laser frequency of $70 \mathrm{kHz}$. At such LDS parameters the interactive width is $142.46 \mu \mathrm{m}$, this means, the minimum space distance is less than $60 \mu \mathrm{m}$, so that according to the previous metallization conditions (the defect occur in the $M s$ range of from 50 to $60 \mu \mathrm{m}$ ), a metallization defect will occur in this case. With $M s$ more than the above range, the metallization defects not occur, as shown in figure 9 (b). It shows the experimental test performed under the LDS parameters including laser power of $9 \mathrm{~W}$, laser speed of $2200 \mathrm{~mm} / \mathrm{s}$ and laser frequency of $90 \mathrm{kHz}$. As a result it is very important to use suitable LDS parameters to give a minimum distance more than this value to insure perfect metallization process without such defects.

In order to avoid the metallization defect, different types of LDS parameters can be used to produce one width of the circuit line, this can be conducted for example by using the LDS parameters including laser power of $12 \mathrm{~W}$, laser speed of $1000 \mathrm{~mm} / \mathrm{s}$ and laser frequency of $70 \mathrm{kHz}$, for the interior area of the circuit line, while at the boundary of the circuit line, can use another different LDS parameters including, laser power in range of from 3 to $9 \mathrm{~W}$, laser speed $2200 \mathrm{~mm} / \mathrm{s}$ and laser frequency in range of from 110 to $130 \mathrm{kHz}$, under such parameters metallization free of defect can be insure.
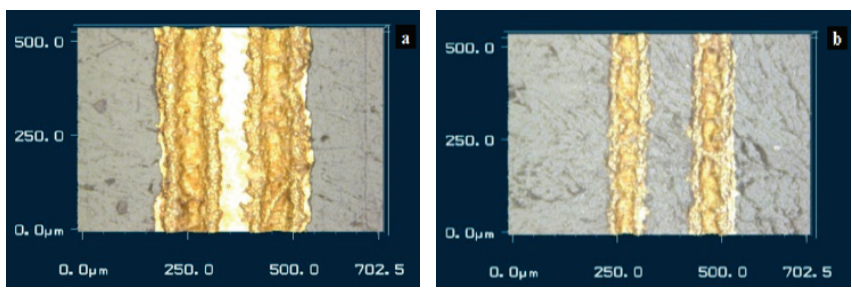

Fig. 9 The metallization process for, (A) Defect, laser power of $12 \mathrm{~W}$, laser speed of $1000 \mathrm{~mm} / \mathrm{s}$ and laser frequency of $70 \mathrm{kHz}$, and (B) No defect, laser power of $9 \mathrm{~W}$, laser speed of $2200 \mathrm{~mm} / \mathrm{s}$ and frequency of $90 \mathrm{kHz}$

Figure 10 shows the relationship among the $M s$, the laser speed and the laser power. It is clear to note that the $M s$ increases with the laser speed, and decreased with the laser power, furthermore the maximum value for the $M s$ of $143.23 \mu \mathrm{m}$ can be achieved under the LDS parameters including laser power of $3 \mathrm{~W}$, laser speed of $2200 \mathrm{~mm} / \mathrm{s}$ and laser frequency of $130 \mathrm{kHz}$ as shown in figure 10 (b). When compare the (Ms) $143.23 \mu \mathrm{m}$ with the Minimum Space distance between two circuit lines can be achieved without defect after the LDS and Metallization process (MSM) including the range of from 50 to $60 \mu \mathrm{m}$, as a result the $M S$ of $143.23 \mu \mathrm{m}$ is more than the $M S M$, in this case the $P S$ can be modified. In fact, the $P S$ value can be changed depending on the two possible conditions below:-

First: $M S-D>M S M$.

In this case the metallization defect will be not occurred, also the value of $P S$ can be reduced with value more than or equal to laser beam diameter D $(65 \mu \mathrm{m}),\left(P s_{\text {new }}=P s-D\right)$. Second: $M S-D \leq M S M$. 
In this case the metallization defect will be occurred, and the $P S$ value is critical and cannot be reduced, $\left(P s_{\text {new }}=P s\right)$. As an example for the case shown in figure 10, the $P S$ is $195 \mu \mathrm{m}$ and the $M s$ is $143.23 \mu \mathrm{m}$. depending on the above two cases $143.23-65=78.3 \mu \mathrm{m}$ which is more than the $M S M$, therefore according to the first condition the $P S$ can be reduced to $P S_{\text {new }}$ of $130 \mu \mathrm{m}$ (195-65).

From figure 10 (a), it can be observed that the minimum value for the $M s$ is $47.5 \mu \mathrm{m}$ can be achieved under the LDS parameters including laser power of $12 \mathrm{~W}$, laser speed of $1000 \mathrm{~mm} / \mathrm{s}$ and laser frequency of $70 \mathrm{kHz}$. This case is matching with the second condition, and gives metallization defects. In order to prevent this type of defect, the Ps must be increased from 195 to $260 \mu \mathrm{m}$, this mean the $M s$ will be increased to $112.5 \mu \mathrm{m}(47.5+65)$.

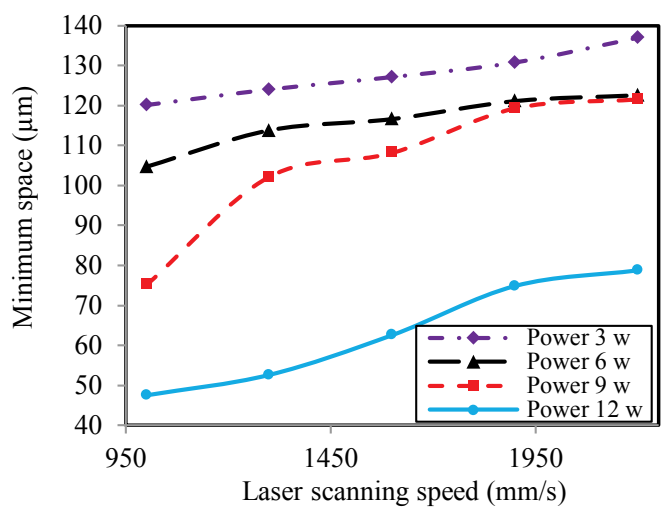

(a) At laser frequency of $70 \mathrm{kHz}$.

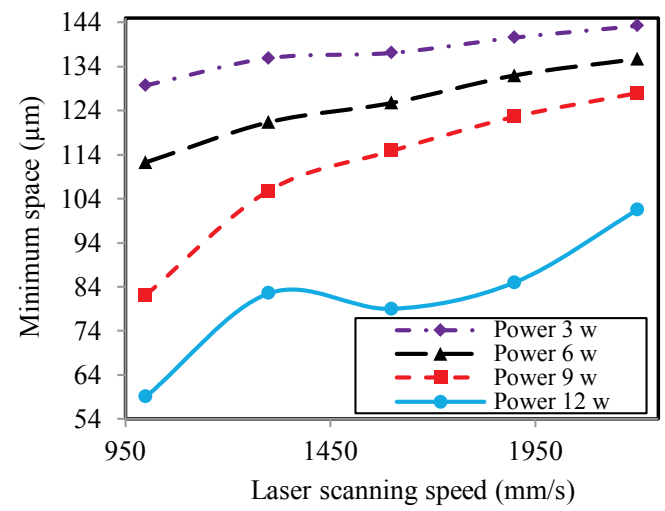

(b) At frequency $130 \mathrm{kHz}$.

Fig. 10 The effect of laser speed on the minimum space distance at different laser power and frequency.

Figure 11 shows the effect of the laser frequency on the $M s$. It can be observed that the $M s$ increases with the laser speed and laser frequency. The $3 \mathrm{D}$ surface for the relationship between the Ms and the LDS parameters is presented in the figure 12 . It can be seen that there are 3 groups of LDS parameters that can be used to produce minimum space distance $125 \mu \mathrm{m}$. The intersection lines between the horizontal plane of $125 \mu \mathrm{m}$ and the surfaces for the laser powers of 3, 6, and $9 \mathrm{~W}$ represent these groups. As mentioned previously the quality of the final product after the metallization process depends on the results of the LDS process, specifically the profile and the dimensions of the groove after the LDS process. Also from this figure, it can be seen that at laser power of $12 \mathrm{~W}$, a Ms with $125 \mu \mathrm{m}$ cannot be produced under the LDS parameters including laser speed in range of from 1000 to $2200 \mathrm{~mm} / \mathrm{s}$ and laser frequency in range of form 70 to $130 \mathrm{kHz}$, while at laser powers of 3 and $6 \mathrm{~W}$ there are many groups for the LDS parameters that can be used to produce the Ms of $125 \mu \mathrm{m}$. Finally at laser power of $9 \mathrm{~W}$, the groups of the LDS parameters are less than that at laser powers of 3 and $6 \mathrm{~W}$. This means, the flexibility for producing a Ms of $125 \mu \mathrm{m}$ at laser powers of 3 and $6 \mathrm{~W}$ more than that at low $9 \mathrm{~W}$ while at laser power $12 \mathrm{~W}$ there is no chance to produce it. As a result it is preferred to use laser power in range of from 3 to $6 \mathrm{~W}$ to produce such Ms.

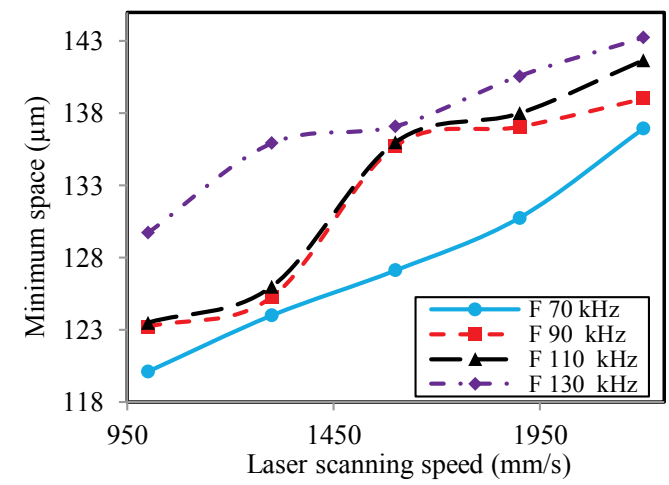

Fig. 11 The effect of laser speed on the minimum space distance at laser power of $3 \mathrm{~W}$ and different laser frequency.

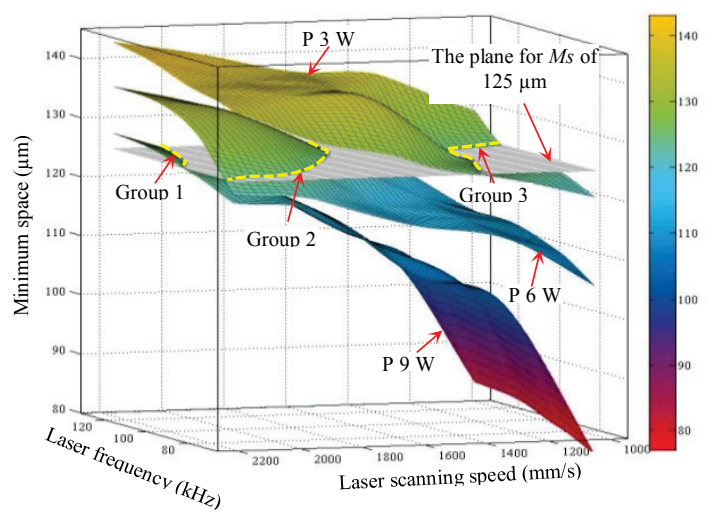

Fig. $123 \mathrm{D}$ surface for the relationship between the minimum space distance and LDS parameters.

\subsection{The effect of LDS parameters on the groove profile}

During the ideal metallization process the metal will cover the entire width of the interactive zone with approximate thickness in rage of from 10 to $12 \mu \mathrm{m}$, with profile depending on the dimensions and profile of the groove before the metallization; this relationship will be presented in figures 13 and 14. Figure 13 shows the 3D microscope laser scan performed under the laser parameters including, laser speed of $2200 \mathrm{~mm} / \mathrm{s}$, laser power of $3 \mathrm{~W}$ and laser frequency of $90 \mathrm{kHz}$, where figure 13 (a), shows the grooves on the polymer surface after the LDS process, while figure 13 (b) shows it after the metallization process. A comparison between these profiles is presented in figure 13 (c). This method can be used to calculate the metallization thickness for the circuit lines on the plate surface. 

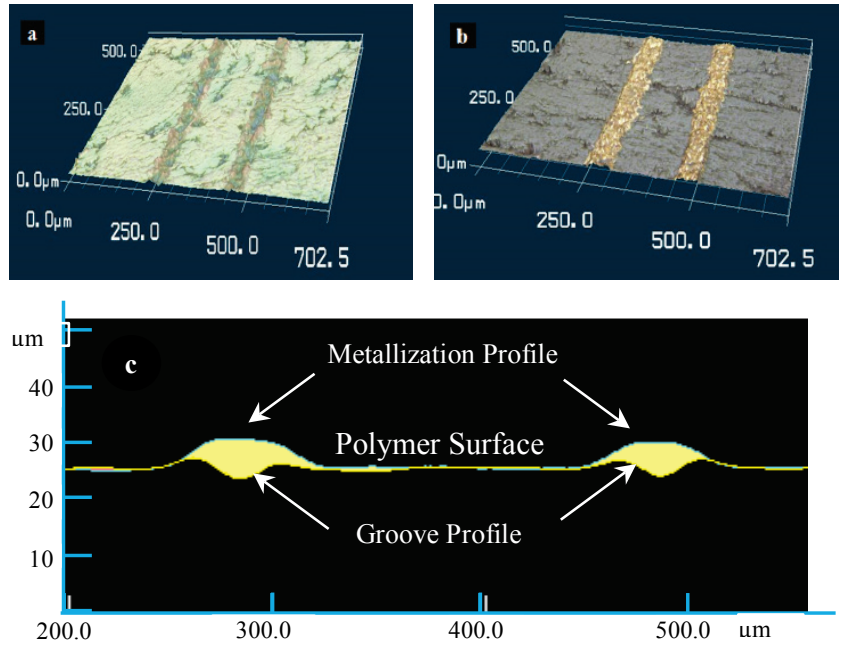

Fig.13 Microscope image for the grooves (a) Before Metallization, b) After Metallization (c) Profile comparison.

Another shape for the metallization profile can be seen in figure 14, for an experimental test conducted under the LDS parameters including, laser speed of $2200 \mathrm{~mm} / \mathrm{s}$, laser power of $9 \mathrm{~W}$ and laser frequency of $110 \mathrm{kHz}$, also this figure the shows a comparison between these profiles before and after the metallization.

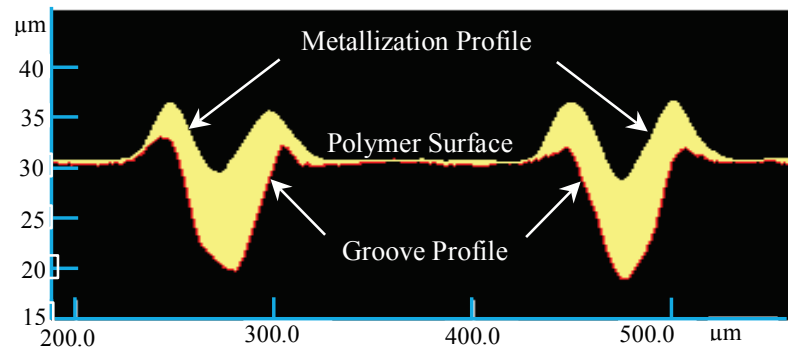

Fig. 14 Profile comparison between the polymer surface before and after the metallization process.

It can observe from figures 13 and 14 that the metallization profile depends on the groove profile; furthermore the groove profile depends on the LDS parameters. As a result the LDS process can be regarded as a key step for the metallization and then the quality of the MID products. Moreover in the above two cases, the metallization thickness varies through the interactive width, maximum thickness in the center of the groove while the minimum at the groove edge. As a result, it is very important to minimize the width of the groove edge as possible as to increase the quality of the metallization and then the quality of the MID products. Figure 15 shows the variation in the metallization thickness under different LDS parameters.

The relationship between the LDS parameters and groove profile must be also investigated. Figure 16, shows the effect of the laser speed on the groove profile. It can be seen from this figure the microscope scans image for the groove under two different laser speed, (a) at the first at laser speed of $1000 \mathrm{~mm} / \mathrm{s}$, and (b) at laser speed of $2200 \mathrm{~mm} / \mathrm{s}$, while the other LDS parameters are the same.

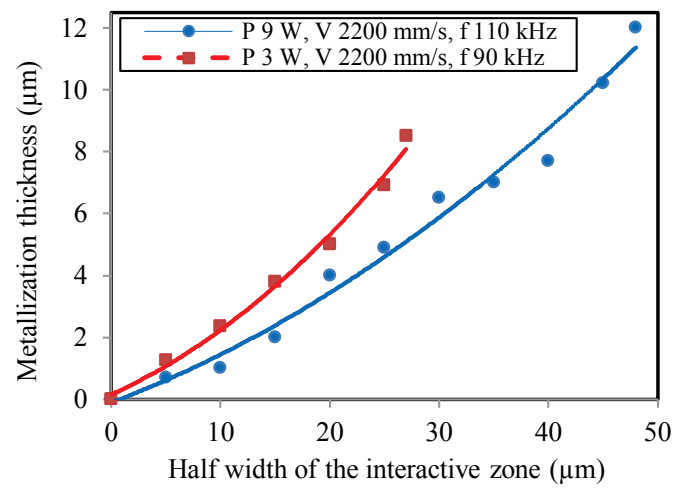

Fig. 15 The thickness of the metallization as a function of LDS parameters.

The comparison between the two groove profiles is shown in figure 16 (c), it can be seen from this figure the effect of the laser speed on the groove profile, dimensions as well as the effect on the groove edge.
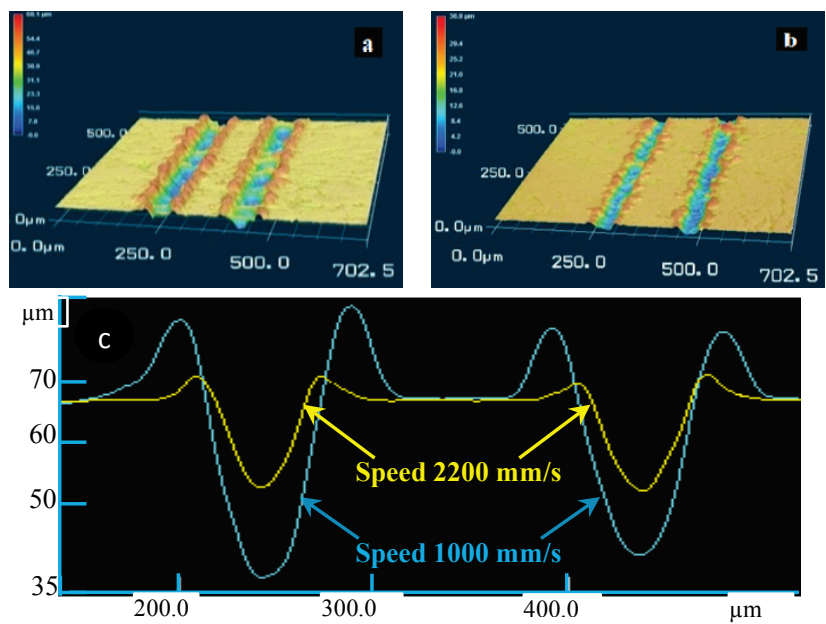

Fig. 16 Microscope image, (a) At laser speed of $1000 \mathrm{~mm} / \mathrm{s}$, (b) At laser speed of $2200 \mathrm{~mm} / \mathrm{s}$, (c) Profile comparison.

The effect of the other LDS parameters including laser power and laser frequency shown in figure 17, where figure 17 (a) shows the comparison between two grooves under two different laser powers of 3 and $12 \mathrm{w}$. While figure 17 (b) shows the profiles for two different laser frequencies of 70 and $130 \mathrm{kHz}$. It can be seen that the effect of the laser frequency is not the same as the laser power and speed. In general, the effect of the laser power and speed on the profile and dimensions are more than laser frequency. Additionally it can be concluded from figures 16 and 17 , that the dimensions of the groove edge namely the width and the height increase with the laser power and frequency and when the laser speed decreases.
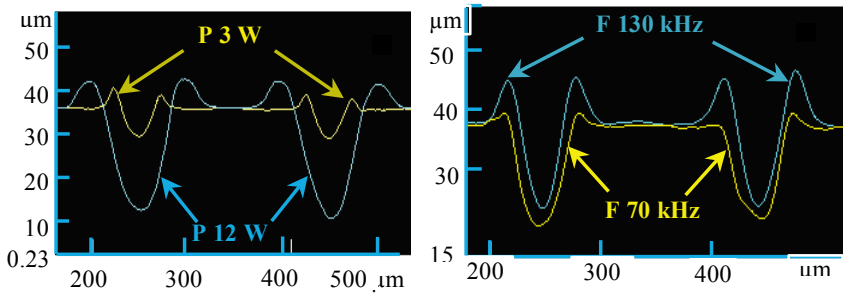

Fig. 17 Comparison between the profiles at (a) Laser powers of 12 and $3 \mathrm{~W}$, (b) Laser frequencies of 70 and $130 \mathrm{kHz}$. 
Figure 18 presents the relationship among the groove edge dimensions and the LDS parameters. It can be seen from this figure that the groove edge dimensions increase with laser power and frequency and decrease with laser speed.

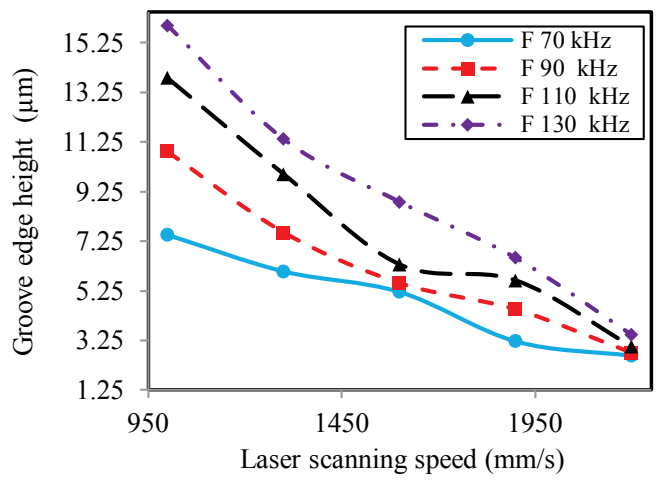

(a) At laser power of $12 \mathrm{~W}$

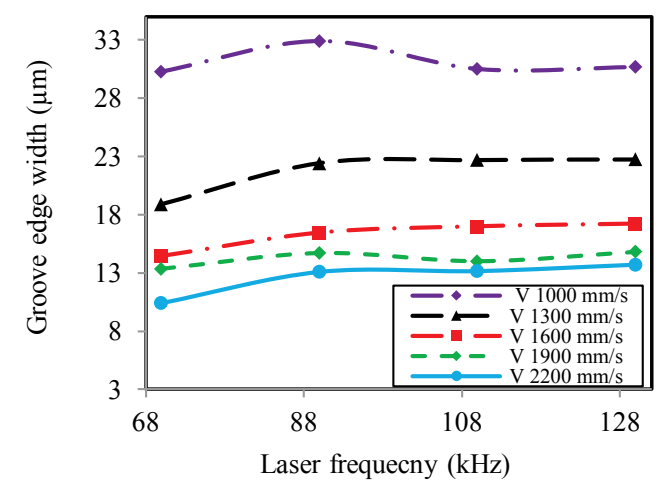

(b) At laser power of $9 \mathrm{~W}$.

Fig.18 The effect of the LDS parameters on the dimensions of the groove edge.

\section{Conclusions}

In this study, the effect of the LDS parameters on the groove dimensions and quality have been investigated, the optimum LDS parameters have been also investigated, and the following points can be concluded.

1-The effect of the laser speed and laser power on the groove profile and dimensions are greater than the effect of the laser frequency.

2-An important phenomenon may be occur in the LDS known as puffiness or blown. This case occurs only under low laser power, high laser speed and high frequency. In such case the amount of the heat flux is high enough to melt the polymer surface but not enough for the vaporization of the molten metal from the surface so that as a result, like puffiness or blown occurs on the plate surface.

3-During the ideal metallization process the metal will cover the entire width of the interactive zone with profile depends on the dimensions and profile of the groove which again depends on the LDS parameters. Moreover, the LDS parameters affect the metallization thickness and quality.

4-For each certain thickness circuit line and minimum space between two circuit lines there are sets of LDS parameters can be used to produce it. The minimum width for the circuit line of $48.5 \mu \mathrm{m}$ can be achieved, while the minimum space distance between two circuit lines can be achieved in the range of from 50 to $60 \mu \mathrm{m}$. The minimum space between two lines can be reduced less than of $50 \mu \mathrm{m}$, but the metallization defects may occur in this case.

5-In order to reduce the metallization defects, the edge dimensions namely the width must be reduced to lowest possible value, because of that the minimum metallization thickness in the edge of the groove. This helps to increase the quality of the metallization and then the quality of the MID products.

\section{References}

[1] J. Franke: "Three-Dimensional Molded Interconnected Devices (3D-MID) Materials, Manufacturing, Assembly, and Application for Injection Molded Circuit Carriers", (Carl Hanser Verlag, München, 2014).

[2] T. Kordass and J. Franke: Galvanic Plating for 3D-MID Applications, Proc. 37th ISSE, on Electronics Technology, Germany, (2014).

[3] B. Bachy and J. Franke, Simulation Of Laser Structuring By Three Dimensional Heat Transfer Model, Proc, XII ICCMSSE, on Materials Science and Surface Engineering, p.1654-1660, Paris, (2014).

[4] L. Romoli1, G. Tantussil and G. Dini1: Layered Laser Vaporization of PMMA Manufacturing 3D Mould Cavities, Annals of the CIRP, Vol.56/1, 209-212, (2007).

[5] G. Raciukaitis and M. Gedvilas, Processing of Polymers by UV Picosecond Lasers, Proc, ICALEO, on Laser Technology, M403, USA, (2005).

[6] E. Spyratou, I. Asproudis, D. Tsoutsi, C. Bacharis, K Moutsouris, M. Makropoulou and A.A. Serafetinides, UV Laser Ablation of Intraocular Lenses: SEM and AFM Microscopy Examination of the Biomaterial Surface, J. Appl. Surf. Sci, Vol. 256, 2539-2545, (2010).

[7] C.R. Mendonca, S. Orlando, G. Cosendey, M. Winkler and E. Mazur, Femtosecond laser micromachining in the conjugated polymer MEH-PPV, Appl. Surf. Sci, Vol.254, 1135-1139, (2007).

[8] R. Torres, T. Kaempfe, M. Delaigue, O. Parriaux, C. Hönninger, J. Lopez, R. Kling and E. Mottay, Influence of Laser Beam Polarization on Laser Micro-Machining of Molybdenum, J. of Laser Micro/Nanoengineering, Vol:8, No:3, 188-191, (2013).

[9] F. Luo, W. Ong, Y. Guan, F. Li, S. Sun, G.C. Lim and M. Hong, Study of micro/nanostructures formed by a nanosecond laser in gaseous environments for stainless steel surface coloring, J. Appl. Surf. Sci, Vol. 328, 405409, (2015).

[10] Evonik Industries, VESTAMID®HTplus LDS 3031 black, Technical Information, Germany, (2014).

(Received: January 15, 2015, Accepted: March 24, 2015) 